\title{
Force measurement on coupled flapping flags in uniform flow
}

\author{
Chuanbao Sun ${ }^{\mathrm{a}}$, SiyinWang ${ }^{\mathrm{b}}$, Laibing $\mathrm{Jia}^{\mathrm{c}}$, Xiezhen $\mathrm{Yin}^{\mathrm{c}}$ \\ ${ }^{a}$ Low Speed Aerodynamic Institute, China Aerodynamics Research \& Development Center, Mianyang, China \\ ${ }^{\mathrm{b}}$ Department of Engineering Structure and Mechanics, Wuhan University of Technology, Wuhan, China \\ ${ }^{c}$ Department of Modern Mechanics, University of Science and Technology of China, Hefei, China
}

\section{Abstract}

An experimental study on the coupled flapping of two identical flags arranged in parallel, tandem, and staggered positions in uniform flow was conducted in a wind tunnel. The dynamic characteristics of each flag were measured using an in-house designed balance and the flapping modes were analyzed through a high-speed video recording technique and a "Spatio-Temporal Evolution" software. For the side-by-side arrangement, the dependences of the kinematic and dynamic parameters on the flow speed and on the mutual distance were observed. The results indicated that the coupling motion and dynamics of two parallel flags in uniform flow were mainly affected by their mutual position. Significant drag reductions were observed when the flags flapped in the in-phase mode for a relatively small separation. For two flags in tandem arrangement, both the upstream and downstream flags experienced a drag reduction compared to the scenario of a single flag in the same flow. Especially, for relatively large separation the upstream flag had a smaller drag coefficient than that of the downstream one. For very small separation, the drag coefficient of the upstream flag was larger than that of the downstream one. Finally, for two flags arranged in a staggered configuration, an anomalous drag distribution was found. This work provides valuable experimental data and casts insight into the coupling mechanism of multiple flexible structures in an air flow.

\section{Keywords}

Force measurement, Flexible flags, Uniform flow, Drag reduction

\section{Introduction}

Fishes and birds increase their efficiency and reduce energy consumption by the synchronized behaviors in groups (Higdon and Corrsin, 1978, Cutts and Speakman, 1994, Weimerskirch et al., 2001). The coupling motion and the drag reduction mechanisms of animals have important significance for bionics applications. It is a great challenge to directly conduct experiments on living animals, due to the difficulty in controlling their movement. Considering the active and 
passive components of animal motions, researchers first studied the passive kinematic and dynamic characteristics by simulating the flexible bodies using various materials.

The coupled flapping of flexible bodies immersed in a flow is a complex 3D problem which can be simplified as a $2 \mathrm{D}$ problem for preliminary study. The coupled kinematics of two side-by-side filaments was firstly investigated by Zhang et al. (2000) using a soap film tunnel. In recent years, it was further studied experimentally (Jia et al., 2007, Wang et al., 2013), simulated numerically using different numerical methods (Zhu and Peskin, 2003, Farnell et al., 2004, Alben, 2009), and analyzed theoretically using a temporal linear instability analysis method (Jia et al., 2007, Shelley and Zhang, 2011). These results showed that two parallel identical filaments exhibited five coupling modes under different flow speeds and separations, namely stretched straight mode (stable mode), in-phase flapping mode, out-of-phase flapping mode, transition mode, and decoupled mode. The amplitude, frequency, and force of the flexible bodies in these modes were quite different. Especially, in a certain condition, the flags in an out-of-phase flapping state usually had a higher frequency than in an in-phase state.

For two tandem flexible bodies in uniform flow, Jia and Yin (2008)systematically discussed their coupled flapping mode and energy distribution through experiments in a soap film tunnel. The results showed that the flapping of the upstream filament was almost unaffected compared to a single filament in the same flow. The flapping amplitude of the downstream filament increased while the frequency was the same as that of the upstream one. The analysis of the results showed that the downstream filament extracted energy from the vortex street and received a larger force than the upstream one. However, for the drag measurement on the tandem flags, Ristroph and Zhang (2008) found a counterintuitive inverted drag relationship, in which the leading flag experienced a significant drag reduction (of up to $50 \%$ ), while the downstream flag suffered a drag increase. This anomalous hydrodynamic drafting was rationalized by dissecting the mutual influence of shape and flow in determining the drag. The experimental setups in the above experiments were quite similar, except that the leading edges of the filaments were completely fixed in the experiments conducted by Ristroph and Zhang (2008) while the leading edges of filaments were fixed in the flow direction but movable in the lateral direction in the experiments conducted by Jia and Yin (2008). Differences in the links of the leading edges of the filaments might be the cause of the result discrepancies. To study the drag reduction of the tandem flags in detail, numerical simulations were conducted on two tandem flexible bodies in uniform flow with different parameters (Zhu, 2009, Kim et al., 2010, Buchak et al., 2010). These numerical simulations indicated that the phase difference of the flag flapping played an important role on the forces acting on each flag. The above mentioned anomalous drag distributions are still in controversy. 
In this paper, we measured the forces on two identical flags when they were arranged in parallel, tandem, and staggered configurations in a wind tunnel, using an in-house designed force measurement system. The coupled kinematics of the flags was analyzed. The flapping mode and force of a single flag were also observed to study the drag reduction mechanism.

\section{Experimental setup}

Fig. 1 is a sketch of the experimental setup and the supporting system of the flags. The experiments were conducted in a low speed wind tunnelwith a section area $1.0 \mathrm{~m} \times 1.0 \mathrm{~m}$ in the Testing Center of Engineering and Materials in University of Science and Technology of China. This wind tunnel is designed for open and closed test sections and the open one was used in our experiments. The stable running speed of this tunnel ranges from $3 \mathrm{~m} / \mathrm{s}$ to $50 \mathrm{~m} / \mathrm{s}$ and the turbulence is below $0.08 \%$. In our tests, the wind speed was measured using a portable hot wire anemometer (TSI-8384-M-GB). For each arrangement of the flags, the flow speed $U$ was continuously increased from $8 \mathrm{~m} / \mathrm{s}$ to $13 \mathrm{~m} / \mathrm{s}$ in our experiments.
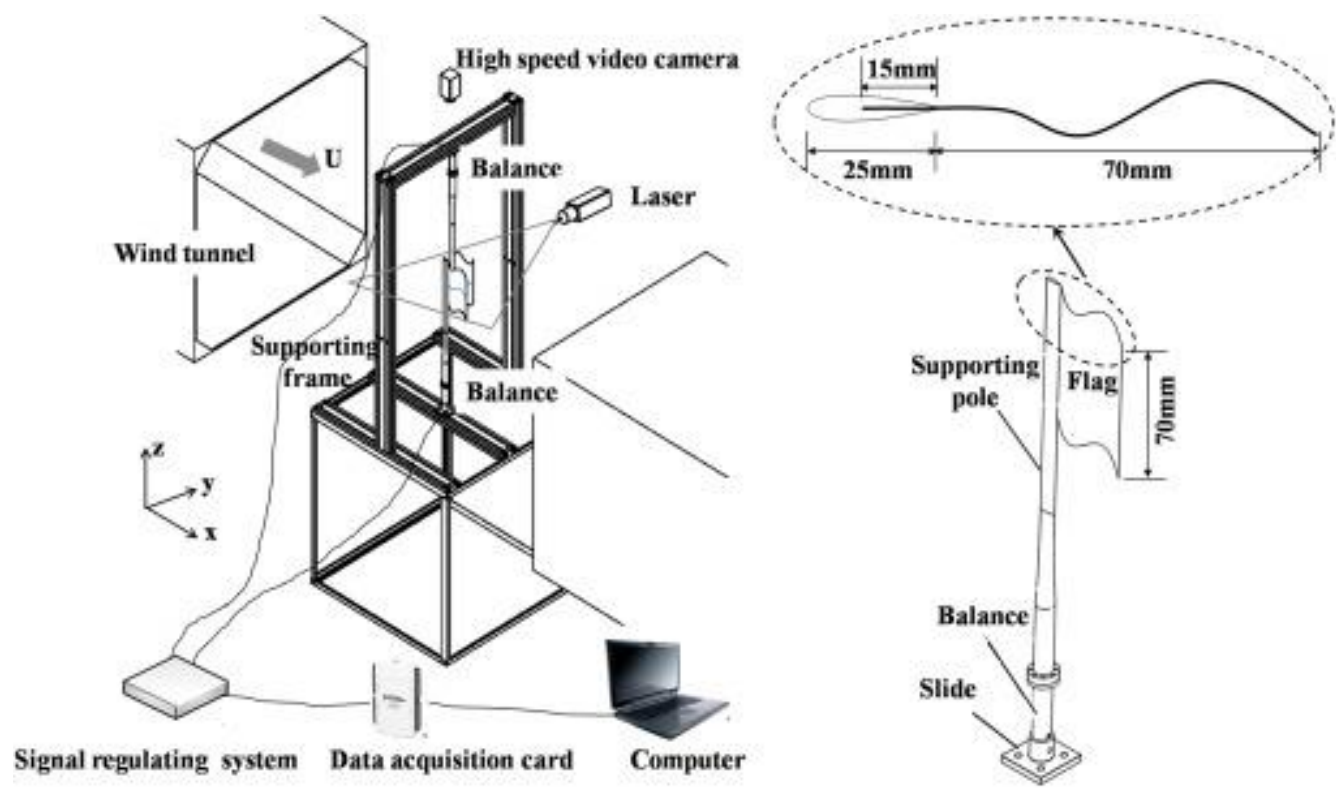

Fig. 1. Sketch of the experimental setup (left) and the supporting system (right).

The flags we studied were squares made of PET (Polyethylene Terephthalate) plastic film. Each of them had an effective area $70 \mathrm{~mm} \times 70 \mathrm{~mm}$. The thickness of the material was $0.15 \mathrm{~mm}$, and its density $\rho_{s}$ and elastic modulus $E$ were measured as $1380 \mathrm{~kg} / \mathrm{m}^{3}$ and $1.8 \mathrm{GPa}$, respectively. As shown in the figure, the leading edge of each flag was held by a supporting pole. The pole was streamlined to decrease its influence on the incoming flow. For the top segment of the pole, the cross section was a NACA0020 airfoil with maximum width $5 \mathrm{~mm}$ and length $25 \mathrm{~mm}$. In this segment of the pole a slot with a length of $15 \mathrm{~mm}$ was present to clamp the leading edge of each flag. For the bottom segment, the cross section was a circular cylinder with a diameter of 
$24 \mathrm{~mm}$, which coincided with that of the balance. The top and bottom segments were joined through a transition segment. The natural frequencies of the supporting pole were $14.7 \mathrm{~Hz}, 19.5$ $\mathrm{Hz}$, and $23.0 \mathrm{~Hz}$ in the lateral, longitudinal and rotational directions. The flapping frequency of the flags was always above $35 \mathrm{~Hz}$, so the influence of resonance was neglected in the results.

Each supporting pole was connected to an in-house designed three-components-balance. The balances had a design load of $30 \mathrm{~N}$ with a measuring accuracy of $0.1 \%$ in each direction (verified by the Laboratory for Balance Testing Technology in China Aerodynamics Research \& Development Center). Voltage signals produced by the balances were sent to a signal processing board (P710, Beijing SinkRenTa Technology Co., Ltd) and then transferred to computers through a data acquisition card (USB-6212, American National Instrument) to obtain force signals.

To observe the kinematic characteristics of the flags, we illuminated the flags using a laser light sheet and recorded the flapping of the flags using a high speed video camera (Speed CAMpro, Weinberger) in the experiments. The image resolution was $1280 \times 512$ pixels and the filming frequency was 1000 frame/s. The flapping frequency of the flags was analyzed using a "Spatio-Temporal Evolution" (STE) software package (Wang et al., 2010), in which strips were cut from each photo taken by the high-speed video camera at a certain position and placed in series on the time axis to get a synthesized image. This synthesized image vividly exhibited the flapping motion of flags at that position like a slit camera. Then the flapping frequency and amplitude were measured from the images.

Choosing the air density $\rho_{f}$, the wind speed $U$, and the flag length $L$ as characteristic scales, denoting the thickness and height of the flag as $d$ and $H$, the main dimensionless influencing parameters in this multiple-structure-fluid coupling problem were defined as: aspect ratio $H^{*}=H / L$, Reynolds number $R e=\rho_{f} U L / \mu$, density ratio $S=\rho_{s} d /\left(\rho_{f} L\right)$, velocity ratio $U^{*}=U\left(12 \rho_{f} L^{3}\right)^{0.5} /\left(E d^{3}\right)^{0.5}$, dimensionless longitudinal gap $G_{x}=\Delta x / L$, dimensionless lateral gap $G_{y}=\Delta y / L$. Here $G$ denoted the gap between the flags, and $\Delta x, \Delta y$ denoted longitudinal and lateral separations as shown in Fig. 2. In this paper, the aspect ratio $H^{*}$ was 1 , the density ratio $S$ was 2.44 , velocity ratio $U^{*}$ was $7.3-11.8$, and $R e$ was $(3.8 \sim 6.1) \times 10^{4}$ under the experimental conditions. 


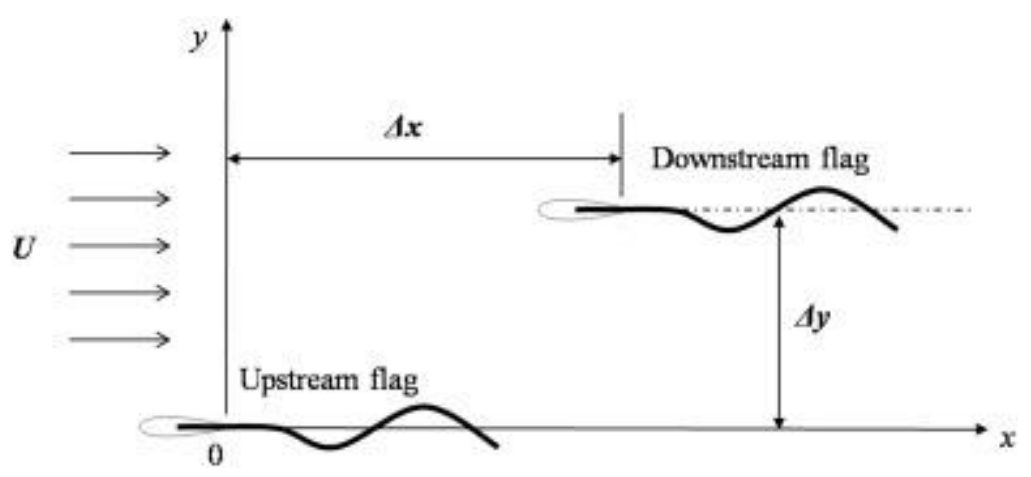

Fig. 2. Arrangement of two flags in uniform flow.

The time-average drag $F_{x}$ was non-dimensionalized as a drag coefficient $C_{d}=2 F_{x} /\left(\rho_{f} U^{2} L H\right)$. For comparison, the kinematic and dynamic parameters were divided by the values in the scenario of a similar flag immersed in the same flow independently. Thus we got the relative frequency $f / f_{0}$, relative amplitude $A / A_{0}$, and relative drag coefficient $C_{d}{ }^{*}=C_{d} / C_{d 0}$. Here $f_{0}, A_{0}$ and $C_{d 0}$ were the frequency, amplitude, and drag coefficient of a single flag in same condition.

\section{Experimental results}

In our experiments, the forces acting on the airfoils with and without flags were measured in the same conditions. Then the actual forces on the flags were calculated as the difference of the two measurements. For comparison, we first conducted experiments on a single flag and studied the dependence of the flapping frequency, amplitude and force on the aspect ratio $H^{*}$, density ratio $S$, velocity ratio $U^{*}$ and Reynolds numberRe. The results showed two phenomena: 1 ) the $3 \mathrm{D}$ effect of the flapping could be ignored when $H^{*} \approx 1$; and 2) $C_{d}$ changed in proportion to the flapping amplitude and increased linearly with $U^{*}$ under the test conditions. The results of two flags in different arrangements are described in the following paragraphs.

\subsection{Side by side configuration}

For two side-by-side flags, the value of $G_{x}$ is zero. As mentioned in the introduction, two parallel flags may act in five different modes in uniform flow. Fig. 3 shows the distribution of coupling modes of two side-by-side flags in $\left(G_{y}, U^{*}\right)$ system in our experiment. 


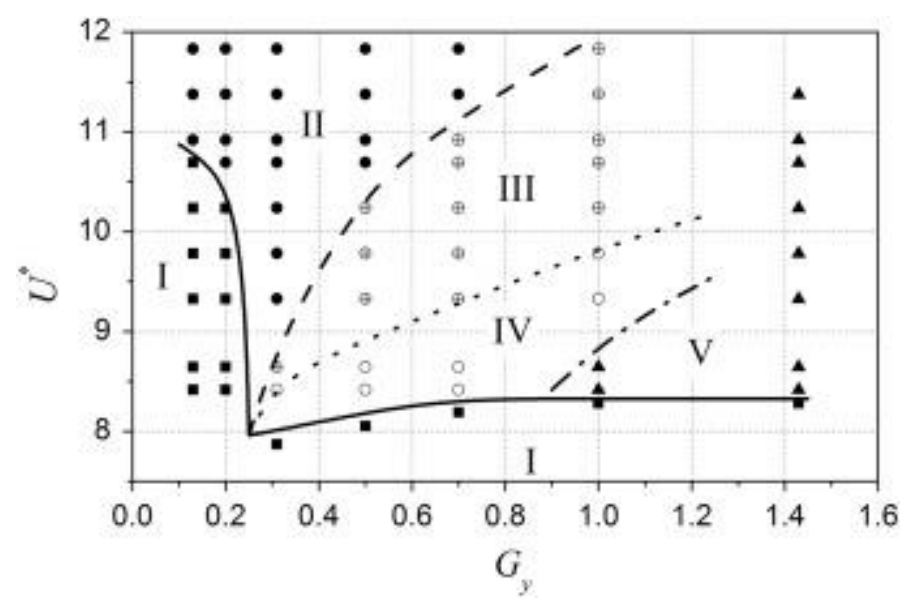

Fig. 3. Coupling mode distribution of two parallel flags in $\left(G_{y}, U^{*}\right)$ space. $\left(G_{x}=0\right.$. $\mathbf{m}$ : I stable mode; $\bullet: I I$ In-phase mode; $\oplus$ :III transition mode; ०: IV out-of-phase mode; $\boldsymbol{\Delta}: \mathrm{V}$ decoupled mode. Solid line — critical boundaries between mode I and other modes, which is also the critical stable boundaries; dashed line — critical oundary between modes II and III; dotted line . . . . critical boundary between modes III and IV; and dash dotted line _. . . critical boundary between modes IV and V).

Wang et al. (2013) studied the coupling mode distribution of two identical flags in wind with $S=1.14, G_{y}=0.1-0.6, U^{*}=4.5-12.5$, and described the out-of-phase, transition, in-phase and quiescent (stable) modes in detail. In our work, the mode distribution was similar to the results in the study of Wang et al. Since the density ratio of flags in our study $S=2.44$ was relatively large, the critical velocity for flags to flap increased compared to that for $S=1.14$ in Wang et al.'s study. For small separation $\left(G_{y}<0.3\right)$, the flags remained straight in stable mode when the flow speed did not exceed the critical velocity $U_{c}{ }^{*}$, and the flags flapped in in-phase mode when $U^{*}$ exceeded $U_{c}{ }^{*}$. The value of $U_{c}{ }^{*}$ for the two-flag case was larger than the critical stable velocity for a single flag in the same fluid $\left(U_{c 0}{ }^{*}=8.46\right)$. This could be due to the fact that the two closely arranged flags always acted as a whole structure and resulted in a better stability than was the case for a single one. For a relatively large separation $\left(0.3 \leq G_{y} \leq 0.7\right)$, the two flags interacted strongly and the stability of the system became weak, which resulted in a smaller $U_{c}{ }^{*}$ than that in the isolated situation. As the flow velocity exceeded the critical value and increased further, the flags would successively flap in out-of-phase, transition, and in-phase modes. For large separation $\left(G_{y}>0.7\right)$, the interaction between the flags weakened as the separation increased and the decoupled mode appeared when the coupling was very weak and the flags were performing independently.

Figures 4, 5 and 6 show the dependences of the relative flapping frequency, amplitude, and average drag coefficient of two parallel flags on their separation in different wind speeds. When two flags interacted at relatively small separation, the relative flapping frequency $f / f_{0}$, the 
amplitude $A / A_{0}$ and the drag coefficient $C_{d}{ }^{*}$ basically increased with the increasing of the lateral separation $G_{y}$. Once the separation was large enough for the decoupled mode to occur, values of $f / f_{0}, A / A_{0}$ and $C_{d}{ }^{*}$ would tend to become unity. In a previous study (Zhang et al., 2000), the flapping frequency of two parallel flags depended much on the coupling mode, and the out-ofphase mode had a frequency 35\% larger than the in-phase mode. Moreover, both the experimental and numerical studies on two parallel flapping flags (Wang et al., 2013) showed that flags in transition mode had two main frequencies. One was comparable to the value of the in-phase mode and the other to that of the out-of-phase mode. However, in our experimental results, the influences of the coupling mode on the flapping frequency f/f0 and amplitude $A / A_{0}$ did not completely agree with the above results as shown in Fig. 4, Fig. 5. For closely placed flags flapping in an in-phase mode, the frequency and the amplitude were smaller than the values of a single flag in the same flow. Moreover, each flag was subjected to a significantly reduced average drag coefficient $(<60 \%)$ as shown in Fig. 6. For relatively large separation, the interaction between the flags weakened and the transition, out-of-phase, and decoupled modes occurred in succession. The dependences of the kinematic and the dynamic parameters on the coupling mode, the arranging distance, and the flow speed are not completely clear, and thus further experiments with wider parameter ranges may be necessary.

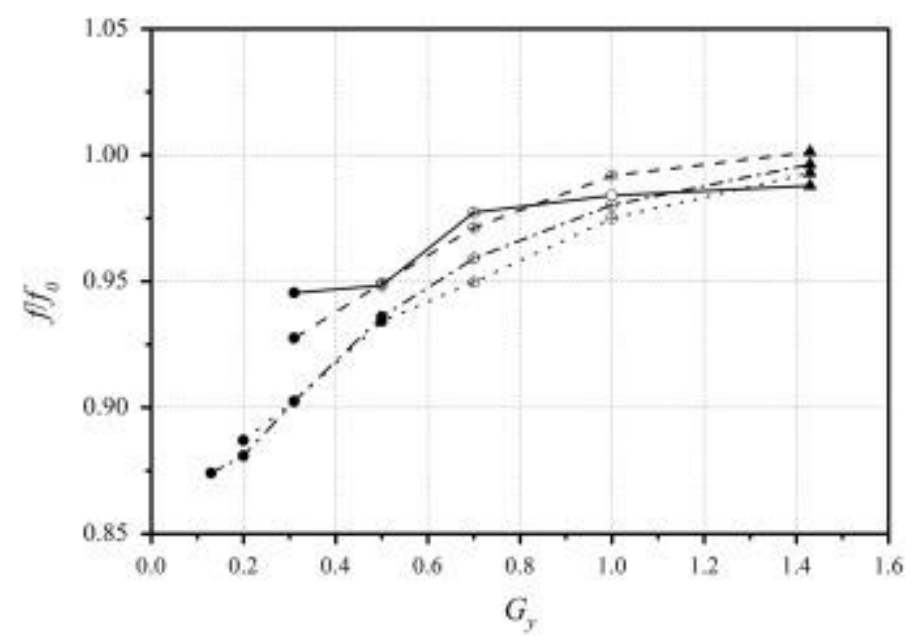

Fig. 4. Dependence of the relative frequency of two parallel flags on the separation. (m: I stable mode; $\bullet$ :II in-phase mode; $\oplus$ :III transition mode; ०: IV out-of-phase mode; $\boldsymbol{\Delta}: \mathrm{V}$ decoupled mode. Solid line: $U^{*}=9.78$; dashed line: $U^{*}=10.24$; dotted line: $U^{*}=10.69$; and dash dotted line: $U^{*}=10.92$ ). 


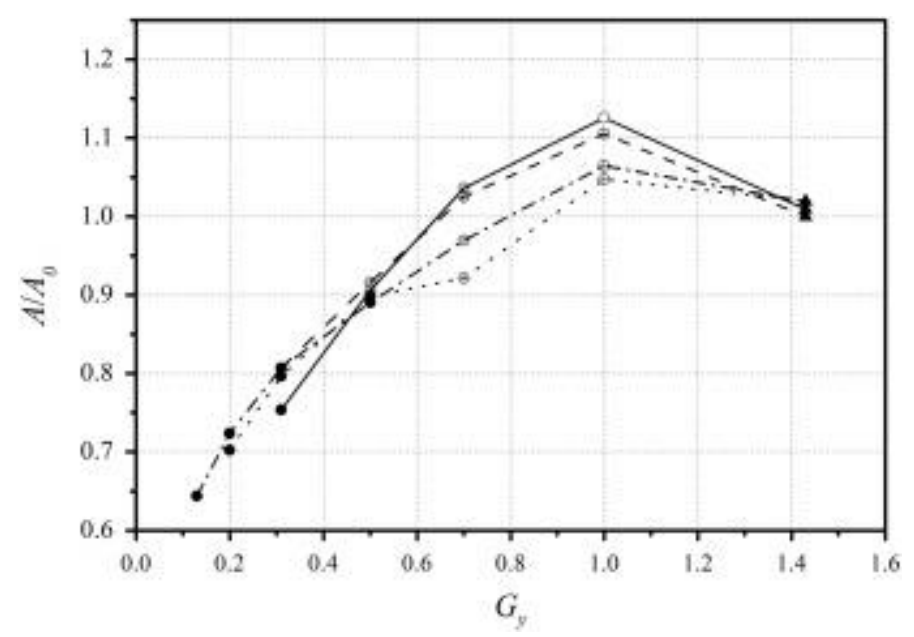

Fig. 5. Dependence of the relative amplitude of two parallel flags on the separation. (m: I stable mode; $\bullet: I I$ in-phase mode; $\oplus:$ III transition mode; ०: IV out-of-phase mode; $\boldsymbol{\Delta}: \mathrm{V}$ decoupled mode. Solid line: $U^{*}=9.78$; dashed line: $U^{*}=10.24$; dotted line: $U^{*}=10.69$; and dash dotted line: $U^{*}=10.92$ ).

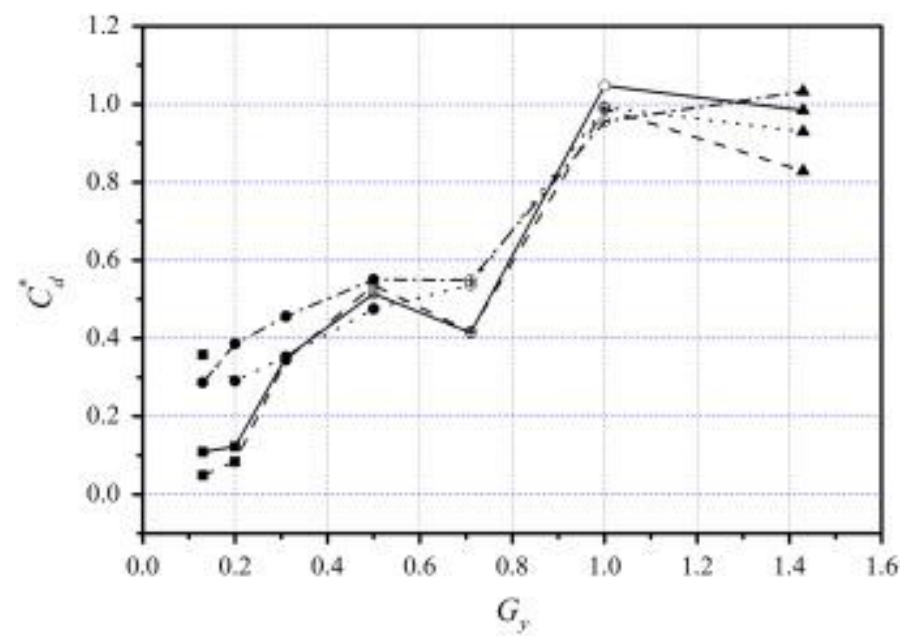

Fig. 6. Dependence of the relative drag coefficient of two parallel flags on the separation.

(匹: I stable mode; $\bullet: I I$ in-phase mode; $\oplus$ :III transition mode; ○: IV out-of-phase mode;

$\boldsymbol{\Delta}:$ V decoupled mode. Solid line: $U^{*}=9.78$; dashed line: $U^{*}=10.24$; dotted line: $U^{*}=10.69$; and dash dotted line: $U^{*}=10.92$ ).

\subsection{Tandem configuration}

The coupling mechanism and force distribution of multiple tandem flags in fluid flow is not yet clear. For tandem arrangement $\left(G_{y}=0\right)$ in our experiments, the two flags flapped in the wind in a coupled mode. The flapping frequencies of both flags were comparable to the value of a single flag in the same flow. The flapping amplitude of each flag in this arrangement changed in proportion to its average drag. 
It is commonly known that for aggregates of rigid objects moving in uniform flow, the objects downstream of the leader generally experience a reduced drag force. However, the interactions of deformable bodies in the fluid flow are poorly understood. Fig. 7 shows the dependences of the relative average drag coefficients of the two tandem flags on the separation distance measured in our experiments. It shows that the average drag coefficients of both the upstream and downstream flags were reduced comparing to that of an identical flag flapped in the same wind flow. In our experiments of relatively large separation $\left(G_{x} \geq 1.5\right)$, the drag coefficient values of both flags increased to the value of a single flag when the separation increased, and the upstream one always had a smaller value. While for flags arranged in line at a small separation $\left(G_{x}<1.5\right)$, the drag coefficient of the upstream flag was larger than that of the downstream one.

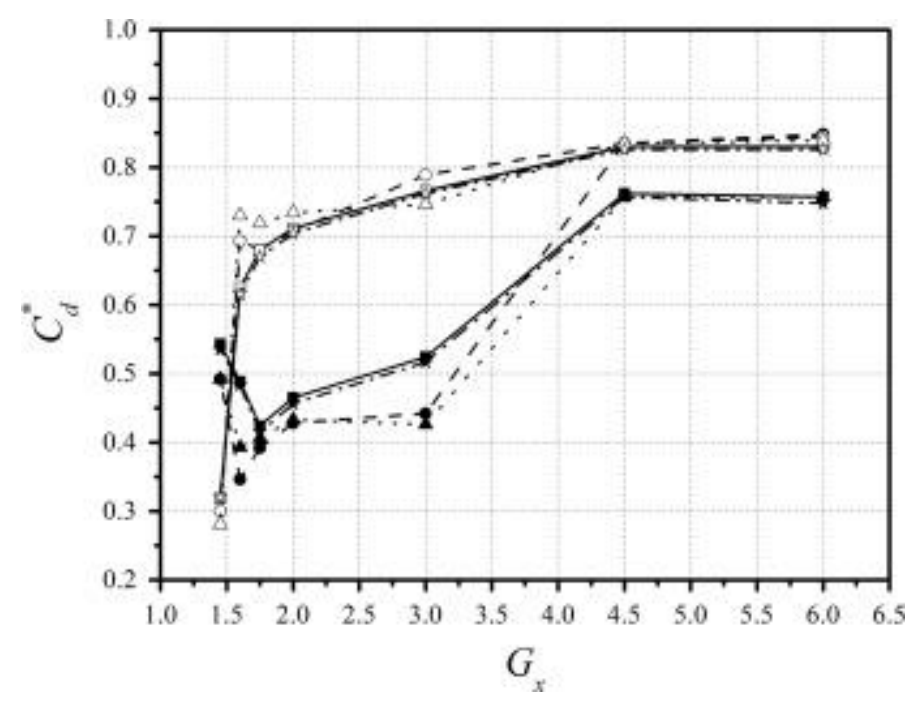

Fig. 7. Dependences of the drag coefficients of two tandem flags on the separation. (Full symbols denote the relative drag coefficient values measured on the upstream flag and empty symbols denote values of the downstream flag. Squares and solid line: $U^{*}=9.78$; circles and dashed line: $U^{*}=10.24$; up triangles and dotted line: $U^{*}=10.69$; and stars and dash dotted line: $U^{*}=10.92$.).

The drags of two tandem flags in uniform flow have also been studied experimentally and numerically in recent years. Ristroph and Zhang (2008) measured the drags of two tandem filaments in flowing soap film, and found that it was the leader of a group who experienced a significant drag reduction (of up to 50\%), while the downstream flag suffered a drag increase. Zhu (2009) simulated the interaction of a pair of tandem flexible flags in a flowing viscous incompressible fluid at lower Reynolds numbers $(40<R e<220)$ using an immersed boundary method. The numerical results showed that when $R e$ and separation $G_{x}$ were large enough so that the flapping of the two flags was self-sustained, the leading flag had less drag than the trailing one; when $R e$ and $G_{x}$ were small enough so that the flags maintained two nearly static 
line segments aligned with the mainstream flow, the downstream flag had less drag than the leading one. The transitional range of $R e$ and $G_{x}$ separating the two different conditions depended on the value of the dimensionless bending rigidity. Kim et al. (2010) modeled two tandem flexible flags in viscous flow at Reynolds number $R e=200$, 300, and 400 using an improved version of the immersed boundary method. In their study, the flexible flapping flag and the vortices produced by the upstream flag were found to interact via either a constructive or a destructive mode. These interaction modes caused significant differences in the drag force acting on the downstream flag. The constructive mode increased the drag force, while the destructive mode decreased the drag force. The occurrence of the two modes depended on both the Reynolds number and the arranged separation. Our experimental results were basically consistent with the literature results despite some differences because of the different parameters of the material and the flow.

\subsection{Staggered configuration}

Figure 8 shows typical maps of the drag coefficients for two flapping flags in various arrangements in moving flow. Here, one flag was fixed at the origin point while the second flag was placed at 44 different positions for the force measurements. The drag coefficient was calculated and normalized by the corresponding values of a single flag in the same flow condition. The points of $G_{x}=0$ represented values of flags in the side-by-side arrangement; points of $G_{y}=0$ represented those in the tandem arrangement; and points of $G_{x} \neq 0$ and $G_{y} \neq 0$ represented those in the staggered arrangement. It should be noted that the data for $G_{y} \geq 0$ in these maps were obtained in our experiments and those for $G_{y} \leq 0$ were obtained by mirror imaging. Data for cases in which the flags collide (red strips) were omitted. 
a

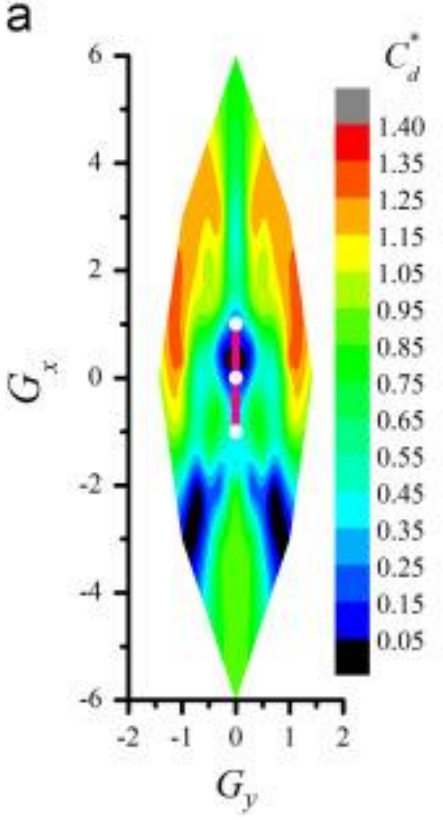

b

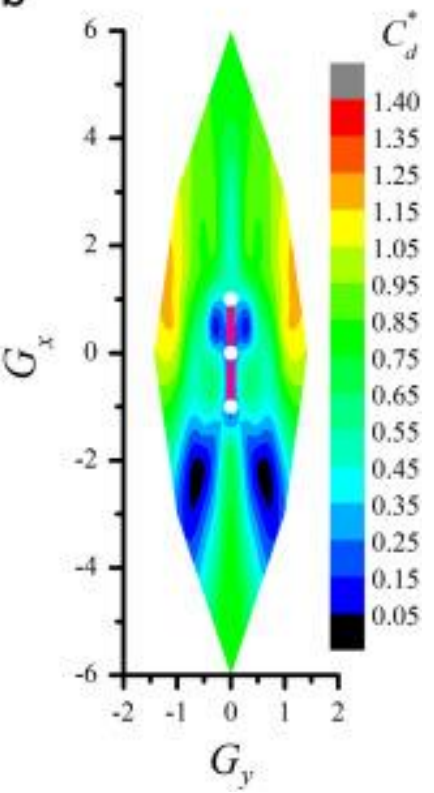

Fig. 8. Maps of relative drag coefficients for two flags arranged in uniform flow ((a) $U^{*}=9.78$; (b) $U^{*}=10.92$. The contours denote values of the relative drag coefficients of the second flag arranged in different positions. The three white dots denote special positions for the flag: upstream, origin point, and downstream. It should be noted that the data of $G_{y} \geq 0$ in these maps were obtained in our experiments and those of $G_{y} \leq 0$ were obtained by mirror imaging. Data for cases in which the flags collide were omitted).

From these plots, we can see a drag reduction for the flag placed slightly upstream ( $0<\mathrm{Gx}<1.0$ and $|\mathrm{Gy}|<0.5)$ of another one. This is consistent with the test results of two flapping filaments in soap film measured by Ristroph and Zhang (2008). When placed laterally upstream, the flag experienced a drag increase (see data for $0<\mathrm{Gx}<3.5$ and $0.5<|\mathrm{Gy}|<1.0$ ). Moreover, when the flag was placed laterally downstream, a drag reduction was measured in our experiment (see data for $-1.5<\mathrm{Gx}<-4.0$ and $0.5<|\mathrm{Gy}|<1.0$ ). In the experiments conducted by Ristroph and Zhang (2008), the flexible body suffered a drag increase at this position. Kim et al. (2010) conducted a numerical simulation study of the coupled flapping of two flexible flags in viscous flow with various parameters, and also found that the drag on the downstream flexible body decreased within certain ranges of Reynolds numbers and mutual distance. In natural observation, fish schools have a diamond shape, dolphins like to swim alongside a moving ship, baby whales always swim in a rear lateral position behind a mature whale. These phenomena suggest the positive biological significance of the drag reduction of flexible bodies moving in a flow in a coupled configuration. 


\section{Conclusions}

This article presented the experimental results on the flapping modes and dynamic characteristics of two side-by-side, tandem and staggered flexible flags in uniform flow. The results were compared with the scenario of a single flag in the same flow. The drag maps of two flags in different arrangements and various flow velocity conditions were presented. The main experimental results demonstrate the existence of 5 different modes for two side-by-side flags under different flow velocities, including stable, in-phase, out-of-phase, transition, and decoupled modes. Generally, the coupled flapping amplitude and drag coefficient of two parallel flags increased with increasing arrangement distance. For two tandem flags, both flags suffered a drag reduction. The drag coefficient of the upstream flag was greater than that of the downstream one when the distance was very small $\left(G_{x}<1.5\right)$, while the situation was opposite when the distance was relatively large $\left(G_{x} \geq 1.5\right)$. For two staggered flags, the drag coefficient of the downstream flag significantly decreased and the upstream one increased when they were arranged with $-1.5<G_{x}<-4.0$ and $0.5<G_{y}<1.0$.

As reported in early studies, the coupling mode and dynamic characteristics of two flexible bodies in uniform flow depend much on the material parameters of both the fluid and the solid structure, as well as on the flow speed, the arrangement, and the separation distance. This paper mainly studied the influence of the flow velocity and of the mutual arrangement in a certain range. To better understand the coupling mechanism, more experimental studies with wider parameter ranges should conducted, together with further numerical and theoretical studies. These will be the main directions of our future work.

\section{Acknowledgments}

The financial supports of the National Natural Science Foundation of China (11102027 and 51479007), the Natural Science Foundation of Hubei Province (2015CFA026), and the Fundamental Research Fund for State Public Beneficial Scientific Institutes of Changjiang River Scientific Research Institute (CKSF2015026/SL) are gratefully acknowledged.

\section{References}

S. Alben Wake-mediated synchronization and drafting in coupled flags. J. Fluid Mech., 641 (2009), pp. 489-491

P. Buchak, C. Eloy, P.M. Reis The clapping book: wind-driven oscillations in a stack of elastic sheets. Phys. Rev. Lett., 105 (2010), p. 194301

C.J. Cutts, J.R. Speakman Energy savings in formation flight of pink-footed geese. J. Exp. Biol., 189 (1994), pp. 251-261

D.J.J. Farnell, T. David, D.C. Barton Coupled states of flapping flags. J. Fluids Struct., 19 (2004), pp. 29-36 
J.J.L. Higdon, S. Corrsin Induced drag of a bird flock. Am. Nat., 112 (1978), pp. 727-744

L.B. Jia, F. Li, X.Z. Yin, et al. Coupling modes between two flapping filaments. J. Fluid Mech., 581 (2007), pp. 199-220

L.B. Jia, X.Z. Yin Passive oscillations of two tandem flexible filaments in a flowing soap film. Phys. Rev. Lett., 100 (2008), p. 228104

S. Kim, W.X. Huang, H.J. Sung Constructive and destructive interaction modes between two tandem flexible flags in viscous flow. J. Fluid Mech., 661 (2010), pp. 511-521

L. Ristroph, J. Zhang Anomalous hydrodynamic drafting of interacting flapping flags. Phys. Rev. Lett., 101 (2008), p. 194502

M. Shelley, J. Zhang Flapping and Bending Bodies Interacting with Fluid Flows. Annu. Rev. Fluid Mech., 43 (2011), pp. 449-465

S.Y. Wang, F.B. Tian, L.B. Jia, et al. Secondary vortex street in the wake of two tandem circular cylinders at low Reynolds number. Phys. Rev. E, 81 (2010), p. 036305

S.Y. Wang, W.G. Duan, X.Z. Yin Transition mode of two parallel flags in uniform flow. Chin. Phys. Lett., 30 (2013), p. 110502

H. Weimerskirch, J. Martin, Y. Clerquin, et al. Energy saving in flight formation. Nature, 413 (2001), pp. 697-698

J. Zhang, S. Childress, A. Libchaber, et al. Flexible filaments in a flowing soap film as a model for one-dimensional flags in a two-dimensional wind. Nature, 408 (2000), pp. 835-838

L.D. Zhu, C.S. Peskin Interaction of two flexible filaments in a flowing soap film. Phys. Fluids, 15 (2003), pp. 1954-1960

Ld Zhu Interaction of two tandem deformable bodies in a viscous incompressible flow. J. Fluid Mech., 635 (2009), pp. 455-475 